\title{
Artelogie
}

Recherche sur les arts, le patrimoine et la littérature de l'Amérique latine

1 | 2011

Brésil, questions sur le modernisme

\section{Du poète au romancier : Blaise Cendrars et le Brésil}

\section{Anita Pires-Saboia}

\section{OpenEdition}

Journals

Édition électronique

URL : https://journals.openedition.org/artelogie/8761

DOI : 10.4000/artelogie.8761

ISSN : 2115-6395

Éditeur

Association ESCAL

\section{Référence électronique}

Anita Pires-Saboia, «Du poète au romancier : Blaise Cendrars et le Brésil », Artelogie [En ligne], 1 |

2011, mis en ligne le 07 octobre 2020, consulté le 07 janvier 2022. URL : http://

journals.openedition.org/artelogie/8761 ; DOI : https://doi.org/10.4000/artelogie.8761

Ce document a été généré automatiquement le 7 janvier 2022.

Association ESCAL 


\title{
Du poète au romancier : Blaise Cendrars et le Brésil
}

\author{
Anita Pires-Saboia
}

\section{NOTE DE L'ÉDITEUR}

Ce texte a déjà été publié :

Colloque International "Voyageurs et images du Brésil"

MSH-Paris, le 10 décembre 2003

Table 4 - Le voyage romantique : littérature et aventure

Disparition d'Anita Clémens Saboia

Anita était arrivée à l'EHESS en 1995 après une carrière particulièrement riche d'expériences variées. Ses études d'espagnol et de lettres modernes l'avaient naturellement conduite à travailler dans des établissements du second degré, mais elle s'était ensuite expatriée au Brésil où elle avait été traductrice à la présidence de la République et membre du jury du prestigieux concours Rio Branco recrutant les diplomates brésiliens. Elle y avait acquis une nouvelle culture qui l'avait profondément marquée. À son retour en France, ses compétences linguistiques - elle était une parfaite quadrilingue maniant avec la même aisance aux côtés de sa langue maternelle le portugais, l'espagnol ou l'anglais - lui avaient ouvert la possibilité de rejoindre le Centre de Recherches sur le Brésil contemporain où elle assurait de nombreuses missions de veille scientifique et de documentation internationale $:$ banque des thèses soutenues en France sur le Brésil depuis le XIXe siècle, bibliothèque bilingue en ligne Mario Carelli, etc. Elle était aussi une cheville ouvrière des Cahiers du Brésil contemporain dans lesquels elle assurait de nombreuses traductions et révisions. Sa sensibilité littéraire l'avait conduite à travailler sur les auteurs français ayant séjourné au Brésil (Cendrars était son préféré) et à traduire de la poésie brésilienne pour les éditeurs français mais aussi à analyser d'un point de vue sociologique les relations intellectuelles entre la France et son pays d'adoption. Récemment, elle s'était 
pleinement impliquée dans la politique linguistique d'aide aux étudiants étrangers de l'EHESS et ses groupes de soutien à l'écriture de la thèse des doctorants hispanophones ou lusophones étaient particulièrement recherchés. Sa brutale disparition nous laisse une profonde tristesse et le regret de n'avoir pas eu le temps de continuer à profiter de tout ce qu'elle nous apportait.

1 Il existe un malentendu entre Cendrars et le Brésil à qui le poète doit sa "naissance au roman, à la prose ». Connus aujourd'hui d'un nombre réduit de spécialistes, ses textes sont encore peu traduits outre Atlantique.

2 Le Brésil «où le simple fait d'exister est un véritable bonheur »', habite pourtant l'œuvre du romancier pendant plus de trente ans. Il y fait figure de grand personnage, à la fois réel et imaginaire, présent dans tous les grands écrits comme dans les pages mineures.

3 Les séjours brésiliens alimentent régulièrement la matière littéraire d'impressions nouvelles, de projets sans cesse renouvelés et divers.

Quand les voyages se terminent ce sont les relations à distance qui alimentent l'inspiration, informent l'écriture. La distance à la fois spatiale et temporelle va lui permettre de créer un nouvel espace fait de mots, d'idées, d'impressions parfois contradictoires qui oscillent entre rêve et désenchantement et cela à travers une grande variété de formes - roman, récit autobiographique, nouvelle, reportage - et dans une langue où l'écrivain converti à la prose n'oublie pas les enseignements du poète.

5 Le Brésil a d'abord joué le rôle de relance créatrice et ce à plusieurs titres : comme pays de tous les possibles, comme pays à la recherche de son identité entre modernité industrielle et mélange des cultures, à la recherche des pères fondateurs : Cendrars a connu Sérgio Buarque de Holanda en qui il voyait un nouveau Radiguet, il a suivi la rédaction de «Retrato do Brasil »². Il lira Gilberto Freyre. Dès son premier voyage, en 1924, il encourage le retour des Brésiliens vers leurs racines, communique à ses amis modernistes son intérêt pour la culture et les rites afro-brésiliens, leur fait redécouvrir l'Aleijadinho, lors d'un voyage au Minas, devient un pro du chorinho.

6 Son premier séjour au Brésil, de février à août 1924 a lieu pour Cendrars, à un moment crucial de sa propre histoire littéraire et de celle de la littérature française. La Grande Guerre a opéré une cassure, en Europe surtout, et on règle ses comptes avec tout ce qui existait auparavant, même avec des gloires encore fraîches comme celles de Cendrars ou même d'Apollinaire.

7 Le Brésil a assuré une reconnaissance littéraire à Cendrars à ce moment-là. Il s'est encore essayé à la poésie au Brésil avec un recueil "Feuilles de route», écrit plutôt pour faire patienter son éditeur et par amitié pour ses amis modernistes.

8 Jusqu'à présent la poésie moderne est la meilleure illustration de cette science nouvelle appelée à bouleverser toutes les vieilles habitudes de l'histoire et de la critique littéraire ${ }^{3}$.

9 C'est par ces mots qu'il dénonce la pauvreté de la langue traditionnelle face à la complexité du monde moderne et proclame la nécessité de forger une nouvelle langue qui s'intéresse à toutes les autres - peinture, sculpture, photographie, machine.

10 Il est intéressant de constater les concordances entre « Feuilles de route » et le recueil de poèmes « Pau Brasil » (1924) : même fascination chez Cendrars et Oswald de Andrade 
pour les nouveaux langages et les textes anciens, les récits des voyageurs, des découvreurs de mondes auxquels l'un et l'autre empruntent des mots, des phrases à des fins de collage.

11 Toujours est-il que le roman va faire irruption dans la vie de Cendrars à son retour en France. Il vient de subir un choc salutaire au cours de ces sept mois grossis à neuf pour la légende - le temps d'une gestation - et dont il dira : «Je ne trouvais pas une parole. Je me mis à penser vertigineusement ». C'est au Brésil qu'il a fait «l'apprentissage de son métier d'écrivain $»^{4}$.

De ce vertige naissent coup sur coup L'Or, la merveilleuse histoire du Général Johan Sutter en 1925 et Moravagine en 1926.

13 L'Or, en préparation depuis 1910, naît quinze ans plus tard sous inspiration brésilienne. "Si la matière de L'Or est californienne, son écriture est brésilienne " commentera Claude Leroy ${ }^{5}$.

14 Cendrars introduit parfois dans le récit des références discrètes au Brésil, comme des plans hors contexte insérés lors du montage d'un film. On y voit que Sutter apprend plusieurs langues dont le portugais, qu'il boit de la caninha ou que sa propriété en ruines attire des nuées d'urubus. A d'autres moments, par personnage secondaire intercalé, l'auteur fait directement allusion au Brésil en utilisant la technique du collage :

Je n'ai jamais vu nulle part la population de couleur se revêtir d'une façon aussi recherchée qu'au Para. Les négresses et les mulâtresses se font des échafaudages d'une grande dimension, en se plantant des grands peignes d'écaille dans leur laine frisée' et des fleurs et des plumes ${ }^{6} .$.

15 Ce qu'entend Sutter de la bouche d'un compagnon de route est en fait un extrait du récit de F. Biard, Deux années au Brésil ${ }^{7}$ reproduit avec exactitude. Mais c'est dans Moravagine que s'affirme avec force le thème brésilien et une forme d'écriture qui va osciller désormais entre le discours analytique et une forme plus imaginative.

En 1917 Moravagine d'abord intitulé La fin du monde, est censé être terminé. Mais dans une lettre à $\mathrm{Cocteau}^{8}$, Cendrars avoue qu'il doit fignoler encore deux ou trois choses. Le manuscrit progresse un peu durant le séjour au Brésil mais il subit de grandes modifications au point de différer complètement de ce qu'il était au départ. Cendrars y retrace l'itinéraire d'un personnage inquiétant, fou apocalyptique qu'il suit dans ses vagabondages criminels jusqu'au Brésil, dans la forêt amazonienne, non-lieu où il s'égare comme il se perd dans sa folie.

$17 \mathrm{Au}$ roman idéologique de la première partie, mise en scène d'une société construite agitée par les conflits idéologiques, répond l'épisode sud-américain qui est une plongée dans l'imaginaire, la déconstruction sociale, l'impossible retour aux sources.

Cendrars laisse libre cours à ses rêves d'utopie et réinvente à sa manière la préhistoire et l'histoire de l'humanité :

Le monde actuel s'est peuplé de l'Occident vers l'Orient. Le flot des générations humaines a suivi le cours des eaux, de l'ouest vers l'est, attiré par le soleil levant, comme les humbles plantes encore humides et pâles qui se tournaient vers la lumière naissante et s'étendaient de plus en plus à l'est, comme les animaux, les animaux et la grande migration des oiseaux. Le berceau des hommes d'aujourd'hui est dans l'Amérique centrale et plus particulièrement sur les rives de l'Amazone. ${ }^{9}$

19 Parallèlement à cette utopie des origines Cendrars va déployer à partir du premier voyage et à la lumière du Brésil, un discours analytique et démonstratif qui s'efforce de 
décrypter le monde moderne et dont le modèle n'est pas la forêt, lieu de l'imaginaire, mais la fazenda qui allie les modes anciens du patriarcat et l'industrie moderne. C'est ce qui apparaît dans « Le principe de l'utilité ${ }^{10}$. «La métaphysique du café $~^{11}$, relève de la même inspiration. Les plantations de café y sont évoquées avec lyrisme comme le modèle du monde moderne :

Nulle part au monde je fus aussi frappé par la grandeur manifeste d'aujourd'hui et par la beauté immuable de l'activité humaine (...) la monoculture a introduit des méthodes nouvelles qu'aucune théorie classique, qu'aucune idéologie n'avaient su prévoir (...) partout le progrès, la richesse, les transformations matérielles vont de pair avec une progression morale, une évolution rapide de la société et une conception nouvelle de la civilisation, de la démocratie, du citoyen et de ses droits ${ }^{12}$.

Mais au-delà des paysages construits, des réalisations humaines, ce sont les gens qui fascinent Cendrars. Ils sont un certain nombre à parcourir son œuvre après avoir guidé sa découverte du Brésil.

Luiz da Silva Prado, lors d'un entretien avec Monique Chefdor, affirmait : «Vous savez, Blaise ne s'intéressait à rien d'autre qu'aux autres. Il ne parlait ni de lui, ni de son œuvre, ni du pays mais des gens ${ }^{13}$.

Retenons de la galerie cendrarsienne la figure emblématique de Paulo Prado, le mécène, le fazendeiro, l'industriel et le dandy fin lettré dont Manuel Bandeira écrivait «qu'au retour d'un de ses voyages en Europe il ramena Cendrars dans ses bagages. »

Oswald de Andrade et Tarsila do Amaral présentent Cendrars à Paulo Prado à Paris, en 1923. Très vite les deux hommes se trouvent des affinités intellectuelles et affectives; naît une grande amitié qui perdure jusqu'à la mort de l'écrivain au point que Prado devient une présence permanente dans son œuvre, un symbole du Brésil qu'il met en scène. Par lui Cendrars découvre l'histoire du Brésil, sa littérature, ses paysages; il reprend à son compte des réflexions de Prado sur le Brésil, lui en prête d'autres et suit tout au long de sa vie, par l'échange épistolaire, l'évolution du pays.

Paul était mon aîné d'un quart de siècle. C'est l'homme avec qui j'ai poussé l'amitié le plus loin. ${ }^{14}$

25 La différence d'âge n'était en réalité que de 17 ans. S'agit-il d'une exagération simplement poétique ou cette amitié se nourrissait-elle, chez l'auteur, de l'admiration pour un père rêvé ? Padroso, le fazendeiro du Morro Azul ${ }^{15}$, est inspiré d'un personnage réel, Luis Bueno de Miranda, ami de Prado. Mais on retrouve dans la création de son nom celui de Prado et le terme padre (père). Cendrars lui attribue son " apprentissage du métier de romancier» alors qu'il reconnait, dans ses lettres, devoir à Prado sa naissance à une autre écriture.

Prado, encore, est le dépositaire de ses rêves, de ses espoirs sur le Brésil, de ses désirs d'utopie. C'est à lui qu'il présentera Le Corbusier dont il voulait qu'il fût de la commission chargée de choisir l'emplacement de la future capitale brésilienne.

Cette fascination pour les gens d'exception le pousse vers les extrêmes: à côté des grands seigneurs il y a ceux qui «n'en ont pas l'air ", les exclus, les marginaux vers qui il se sent attiré au point de leur ressembler. On se souvient de l'étonnement que son allure suscita chez ses admirateurs venus l'accueillir à Rio.

28 A ce propos, Cicero Dias rapporte un épisode que Paulo Prado se plaisait à raconter : l'arrivée impromptue et rocambolesque chez lui, à São Paulo, de Cendrars et Capistrano de Abreu. Le poète et l'historien se sont retrouvés devant sa porte et ont alors fait 
connaissance. Leur accoutrement, leur visage buriné et fatigué par le voyage en train de Rio à São Paulo ont affolé le portier qui a filé réveiller le maître des lieux pour se plaindre qu'un vagabond et un infirme se prétendant les amis de monsieur fumaient et menaient du tapage devant la porte. L'un d'eux, manchot, paraissait un mendiant de foire. Prado qui savait ses amis en ville reconnut sans peine le poète suisse dans le mendiant et l'historien brésilien dans le vagabond. ensemble parfois contradictoire rapporte tous les espoirs et toutes les déceptions de Cendrars sur le Brésil qui trente ans après le dernier voyage ne répond plus aux rêves d'antan même s'il court toujours derrière le «bluff du modernisme ». secrètes, à l'imaginaire de Cendrars parce qu'il a mis à sa disposition des formes lui permettant de se représenter soi-même et d'orienter sa création littéraire. 
Le Brésil de Cendrars, cette "Utopialand, un pays qui n'appartient à personne ", n'appartient en fait qu'à lui-même et finit par lui échapper. D'où le malentendu peutêtre avec les Brésiliens qui ne s'y reconnaissent pas, mais aussi avec le public français qui a redécouvert Cendrars sans toutefois comprendre l'importance d'une présence brésilienne éloignée de tous les clichés connus. Conscient de ce décalage Sergio Milliet lui rendit hommage lors de sa mort, en 1961 :

L'authenticité de Cendrars ne se trouve pas dans la réalité de ses affirmations mais plutôt dans l'ambiance effective dans laquelle il les vit à tout moment, à tout instant. Avec l'intuition d'un paléontologue qui, de quelques os, reconstitue le squelette d'un animal antédiluvien, notre poète, à l'aide de quelques données et de quelques anecdotes, écrit un roman de notre Pays, et s'il n'est pas tout à fait le nôtre, il pourrait parfaitement l'être. Aussi, quand il nous raconte qu'il a traversé le sertão de Goiás dans son Alfa Romeo, dit-il un mensonge, il invente, mais comme nous sentons que nous sommes effectivement dans l'état de Goiás! Un Goiás qui n'était pas encore ce que disait Cendrars mais qui le serait 20 ans plus tard... ${ }^{17}$

\section{NOTES}

1. Blaise Cendrars, Brésil, des hommes sont venus, éd. Fata Morgana, 1987, p. 18

2. Paulo Prado, Retrato do Brasil, 1928

3. Conférence faite à São Paulo, le 21 février 1924.

4. Blaise Cendrars, « La Tour Eiffel sidérale » in Le lotissement du ciel, Denoël, 1949.

5. Claude Leroy, L'Utopialand de Blaise Cendrars, Actes du colloque de Sao Paulo, 4-7 août 1997, L'Harmattan, 1998.

6. L'Or, CEuvres Complètes, Denoël II, p. 139.

7. Biard, F. Deux années au Brésil, Paris, Hachette, 1862, pp. 336-7, cité par Carlos Augusto Calil, in « Le contrebandier de cigares », Brésil, L'Utopialand de Blaise Cendrars, Actes du colloque de Sao Paulo, 4-7 août 1997, L'Harmattan, 1998.

8. Blaise Cendrars, L'Or, op cit.

9. Blaise Cendrars, Moravagine, Denoël.

10. Publié en revue d'abord puis dans Moravagine, texte qui marque la transition entre l'ancien et le nouveau monde.

11. Aujourd'hui, 1931.

12. Aujourd'hui, 1931, pp. 236.

13. Monique Chefdor, "Cinquante ans après: souvenirs, réminiscences, saudades", in L'Utopialand de Blaise Cendrars, op cit.

14. Bourlinguer, 1948. Cendrars avait l'habitude de franciser les prénoms brésiliens.

15. Le lotissement du ciel, 1949.

16. Blaise Cendrars, Outremer à Indigo, Denoël, 1940.

17. In «Livros de 30 dias : Blaise Cendras ». Anhembi, Ano XI, n 124, março de 1961, p. 366, cité par G. Marcondes Machado, "L'effet d'irréel dans Moravagine", Brésil, l'Utopialand de Blaise Cendrars, l'Harmattan, 1998, pp. 246-247. 


\section{AUTEUR}

ANITA PIRES-SABOIA

Professeur certifiée CRBC/EHESS 